\title{
Comment on invalid JWKB unstable solutions of combined inertial-Rossby waves
}

\section{J. F. McKenzie}

Department of Mathematics, Statistics and Physics, Durban University of Technology, Durban, South Africa

School of Mathematical Sciences, University of KwaZulu-Natal, Durban, South Africa

Correspondence to: J. F. McKenzie (mckenziej@ukzn.ac.za)

Received: 8 February 2012 - Revised: 13 March 2012

In three recent papers (McKenzie, 2009, 2011a, b) on the combined theory of gravity inertial-Rossby waves on a $\beta$-plane it was shown that "local" JWKB solutions exhibit complex roots for the wave frequency if the speed of the low-frequency, long-wavelength westward propagating Rossby wave exceeded that of the higher frequency (KelvinPoincaré) inertial wave speed. If the ratio of the former (Rossby) speed to the latter (inertial) speed is denoted by $m$ (a function of $\beta, f$ the Coriolis parameter and $V$ the inertial speed) the condition for complex roots $m>1$ translates into a region in the parameter space of the latitude of the $\beta$-plane and the rotational Mach/Froude number of the planet. Therefore it is tempting to associate these complex roots in this parameter space with instability of the system arising from the coalescence of modes (Akhiezer et al., 1967), or from the interaction between positive and negative energy waves (Cairns, 1971).

However a note of caution was sounded in the latest paper of the series (McKenzie, 2011b) to the effect that JWKB solutions whose latitudinal wave number remained unconstrained, may not, in fact, provide an acceptable solution to the "global" problem which involves the imposition of latitudinal boundary conditions.

Indeed Paldor (2010) showed that if mid-latitude JWKB type solutions are subject to, for example, rigid wall conditions in one hemisphere, the corresponding eigenvalues precluded the fulfillment of the "instability condition" $m>1$, and therefore that the system was globally stable. Furthermore Paldor emphasized that in the well known case of an equatorial $\beta$-plane, the eigenvalues and eigenfunctions correspond to stable (real) values for the wave frequency. Finally, a global energy invariant for the system (Pedlosky, 1987; Paldor, 2010) demonstrates that, in the absence of shear flow or negative buoyancy, the system is stable.

In summary the latitudinal dependence in the equations arising from the variation of the Coriolis force, which is an essential feature of the Rossby wave, together with appropriate boundary conditions gives rise to an eigenvalue problem instead of a purely local solution with its associated dispersion equations. This eigenvalue problem (whether the $\beta$ plane is centred on mid-latitudes or at the equator) has no unstable solutions. Therefore it is clear that the claim that the system is unstable, based solely upon local JWBK solutions, made by me is invalid.

Acknowledgements. I would like to thank M. E. McIntyre and R. A. Cairns for helpful discussions and J. Pedlosky for enlightening correspondence.

Topical Editor C. Jacobi thanks one anonymous referee for her/his help in evaluating this paper.

\section{References}

Akhiezer, A. I., Akhiezer, I. A., Polovin, R. V., Sitenko, A. G., and Stepanov, K. N.: Collective oscillations in plasma, MIT Press, Cambridge Massachusetts, 1967.

Cairns, R. A.: The role of negative energy waves in some instabilities of parallel flows, J. Fluid. Mech., 92, 1-14, 1971.

McKenzie, J. F.: Instability of coupled gravity-inertial-Rossby waves on a $\beta$-plane in solar system atmospheres, Ann. Geophys., 27, 4221-4227, doi:10.5194/angeo-27-4221-2009, 2009.

McKenzie, J. F.: On the unstable mode merging of gravity-inertial waves with Rossby waves, Ann. Geophys., 29, 1377-1381, doi:10.5194/angeo-29-1377-2011, 2011a.

McKenzie, J. F.: Instability of combined gravity-inertial-Rossby waves in atmospheres and oceans, Ann. Geophys., 29, 997-1003, doi:10.5194/angeo-29-997-2011, 2011b.

Paldor, N.: On spurious instabilities on the $\beta$-planes with no mean flows, Ann. Geophys., 28, 1737-1739, doi:10.5194/angeo-281737-2010, 2010.

Pedlosky, J.: Geophysical fluid dynamics, Springer-Verlag, 1987. 